\title{
Approaching Vehicle Detection Method with Acoustic Analysis using Smartphone for Elderly Bicycle Driver
}

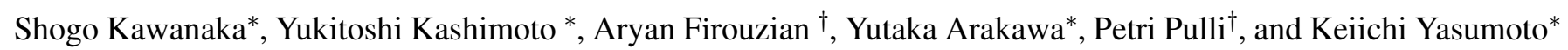 \\ ${ }^{*}$ Graduate School of Information Science, Nara Institute of Science and Technology, \\ Ikoma, Nara 630-0192, Japan, Email: see http://ubi-lab.naist.jp/, http://inet-lab.naist.jp/ \\ $\dagger$ University of Oulu, Pentti Kaiteran katu 190014 Oulu, Japan
}

\begin{abstract}
More than 60 percentage of fatal accidents while riding a bicycle is caused by elderly people over 65 years old. The main cause is the detection delay of approaching vehicle caused by the decrease of cognitive function due to aging. In this paper, we propose an approaching vehicle detection method using a smartphone aiming to support bicycle operation to prevent elderly people from fatal accidents while riding a bicycle vehicle. Among various sensors embedded in a smartphone, we focus on microphone as the most suitable sensor for detecting an approaching vehicle. We collected sound data in a real environment and created an approaching vehicle detection model by using machine learning. Finally, as a result of accuracy evaluation with 10-fold cross-validation, we confirmed that it can detect approaching vehicle with an average F-value of 97.4 [\%].
\end{abstract}

\section{INTRODUCTION}

A bicycle is a convenient means of transportation which can be used even for children and elderly people on a daily basis. In addition, it is reconsidered as eco-friendly transportation way that can cope with environmental problems such as global warming. Furthermore, use of a bicycle is expanding with an increase of health consciousness and great efforts in natural disasters. However, a parentage of bicycle traffic accidents in traffic accidents is increasing, whereas the number of traffic accidents is decreasing. Among them, 60 percentage of fatal accidents is caused by elderly people [1]. We can expect that a number of fatal accidents which are caused by elderly people will be increased with the increase of bicycle use and the number of elderly people in Japan where the population is aging [2]. Therefore, countermeasures against accidents for elderly people while riding a bicycle is imperative.

In consideration of this situation, the Japanese government and each of local governments have been starting to implement countermeasures. One of them is "Tokyo 2020 Project," that is designed for Tokyo 2020 Olympic Games [3]. In this project, maintenance of bicycle traveling space is mentioned, but the enormous cost is required to newly make a bicycle lane. Additionally, large-scale construction is needed in some places or there are inadaptable terrains. Thus, it is difficult to apply such a maintenance everywhere in our living area.

In addition, the number of vehicles having a collision avoidance function has been increasing in recent years. However, the situation is limited or there are still many non-equipped vehicles present.

Another way to prevent accidents is the way bicycle riders take measures. Generally, since cognitive abilities and physical abilities decline due to aging, it is assumed that a delay in danger recognition and avoidance behavior is introduced. We consider that this "delay" is the main factor in traffic accidents in elderly people. Therefore, in order to reduce fatal accidents by elderly people, it is important to support cognitive function by using a notification tool which detects approaching vehicle and notifies the user. Table 1 shows the summary of the pros and cons of each approach to reduce bicycle accidents.

As existing studies on a vehicle detection method, first, Stephen et al. [4] have proposed a notification system that detects an approaching vehicle from behind using a video camera and an embedded computer and notifies the approaching vehicle. Next, Shimoyama et al. [5] have proposed a prevention system that carries out travel analysis using different kinds of sensors, warns the dangerous driving condition before accidents occur and prevents accidents in advance. In these studies, the target users are limited since a camera or sensor devices is needed in these systems. In addition, Yoshida et al. [6] have proposed a proximity notice system based on intervehicle communication. This system assumes that dedicated equipment is installed on both sides, so it is not common nowadays. Given the cost of practical use, it is desirable to have a system that can detect the approaching vehicle only with a device, which is normally owned by the bicycle user.

In this study, we solve these problems with acoustic analysis using the bicycle user's smartphone. Then, we propose a system that detects approaching vehicle by unique noise which is generated when vehicles approach by using a microphone embedded in a smartphone and notifies bicycle user of it.

In this paper, we conducted preliminary experiment for the approaching vehicle detection method to find out which embedded sensors in a smartphone are useful to detect approaching vehicle. As a result, we found that approaching vehicle can be detected by frequency analysis of the ambient sound data obtained from a microphone. Second, we created a data set from ambient sounds collected while riding a bicycle 
TABLE I

SUMMARY OF THE PROS AND CONS OF EACH APPROACH TO REDUCE BICYCLE ACCIDENTS

\begin{tabular}{|c|c|c|}
\hline Approaches & Pros & Cons \\
\hline Improvement of roads & Fundamental solution & $\begin{array}{c}\text { Large cost } \\
\text { Difficult to apply everywhere }\end{array}$ \\
\hline Collision avoidance function of vehicle & Pedestrians need not any devices & $\begin{array}{c}\text { Effective situation is limited } \\
\text { Still many vehicles doesn't equipped }\end{array}$ \\
\hline Countermeasure from bicycle driver & $\begin{array}{c}\text { Easy to introduce } \\
\text { Can be use anywhere }\end{array}$ & $\begin{array}{c}\text { Smartphone is required } \\
\text { Effective situation is limited }\end{array}$ \\
\hline
\end{tabular}

using a smartphone and created a vehicle detection model by machine learning using Weka (REPTree). Finally, as a result of evaluation with 10 -fold cross-validation, we confirmed that the approaching vehicle detection model can detect approaching vehicle with high accuracy, an average F-value of 97.4 [\%].

\section{RELATED WORK}

In this section, we present a brief overview of existing studies. First, Stephen et al. [4] developed a probe bicycle called "Cyber-Physical Bike" that can recognize approaching vehicles and notify the bicycle user by using video camera attached to the saddle of the bicycle in the backward direction and embedded microcomputer. In this study, not only the detection of vehicles but also the risk of collision can be evaluated. However, it is difficult to use this system for general users because the target users are limited since a camera or sensor devices are needed in this system.

Next, Yamashita et al. [5] analyzed the driving situation of the bicycle using multiple kinds of sensors and proposed a notification system that warns when dangerous driving is performed. This system has three sub-systems: traveling condition detection unit, traveling environment detection unit and warning unit, and they need several kinds of sensors or modules. Similarly, this system also can not generally be used since it requires the attachment of many sensors on the bicycle.

Conversely, Takagi et al. [7] proposed a vehicle detection scheme using a smartphone carried by a pedestrian. They exploit a high frequency switching noise generated by a motor unit in electric vehicles(EVs) and hybrid vehicles(HVs). As a result, a J48 classier implemented on the smartphone can tell weather an EV or a HV is approaching or not in the accuracy of $92[\%]$ and $82[\%]$, respectively. However, this scheme aims at only detection of EVs and HVs and the target user is pedestrians, so it does not match with our purpose.

In these previous studies, it is excellent to be able to evaluate the risk of collision and to detect user's dangerous driving. However, it is difficult to put them into practical use since they need dedicated sensors. Thus, in this study, we propose an approaching vehicle detection method which aims to detect all vehicle types using only a smartphone while running bicycle without additional sensors or devices on the bicycle.

\section{PRELIMINARY RESEARCH}

This section describes a preliminary research for vehicle detection using a smartphone, as the first step to develop an approaching vehicle detection method. First, we discuss which sensors are suitable for detection, based on related work. Subsequently, we confirm detection availability of approaching vehicle using them with preliminary experiment.

\section{A. Investigation of Vehicle Detection Method}

As mentioned in Section 2, infrared ray sensor, ultrasonic sensor, geomagnetic sensor, camera and microphone have been used to detect vehicles [4], [5], [9], [10]. Generally, those sensors are embedded on smartphone. However, when we use a camera, it is necessary to always keep recording backward while riding a bicycle and it is not easy to attach it to the rear part of the bicycle. In the case of using an infrared ray sensor which is embedded on smartphone, it is not suitable for our purpose because it can measure only several centimeters and the resolution is not high. Based on these reasons, we focused on geomagnetic sensor and microphone and conducted a preliminary experiment to confirm whether vehicles can be detected using these sensors.

\section{B. Preliminary Experimental Method}

In order to confirm whether vehicles can be detected by using geomagnetic sensor and microphone which is embedded on smartphone, we attached a smartphone on a bicycle with a mounter as shown in Fig. 1 and conducted an experiment on a straight road in a relatively quiet residential area. The experimental course is shown in Fig. 2.

We used Nexus 6 (Motorola, XT1100, Android 6.0) as a smartphone and "AndroSensor ${ }^{1 "}$ " as a sensor data collection application. In addition, a GoPro camera was also attached on a bicycle and took a movie during riding to record a ground truth. Fig. 1 shows placement of the smartphone and GoPro camera. The geomagnetic data and the sound data are acquired with sampling rates of $10[\mathrm{~Hz}]$ and $16.0[\mathrm{kHz}]$, respectively, and the recording format of the sound data is 16-bit linear PCM. At this time, we used a general bicycle without any equipment such as suspension.

\section{Experimental Results and Discussion}

The results of collected geomagnetic data is shown in Fig. 3. The area highlighted in red color represents the vehicle approaching period. In this graph, changes are seen when a vehicle is approaching, however, significant changes are also

\footnotetext{
${ }^{1}$ http://www.fivasim.com/androsensor.html
} 


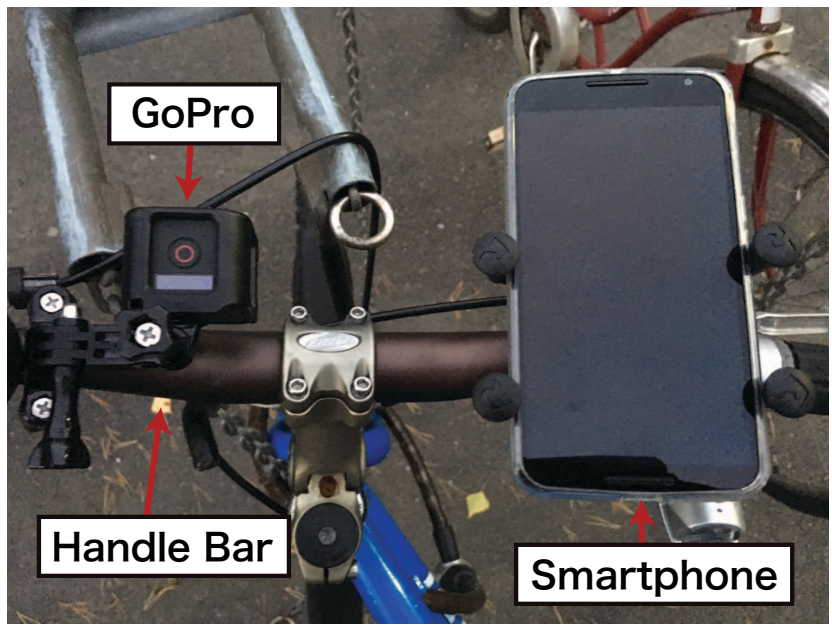

Fig. 1. Placement of Smartphone and GoPro Camera

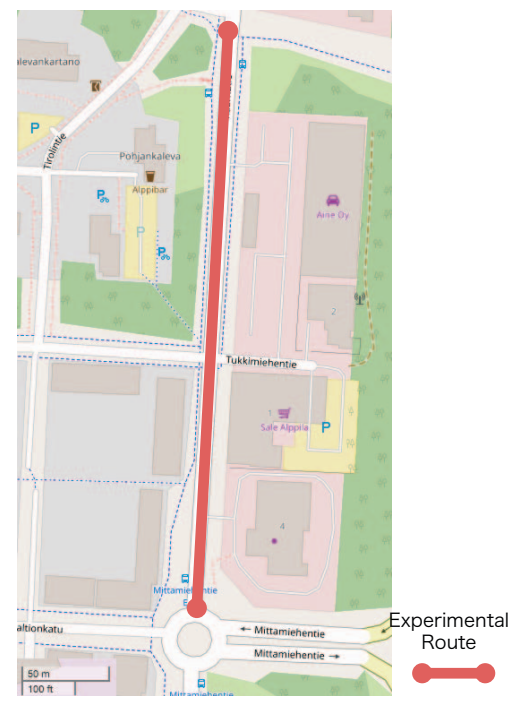

Fig. 2. Experimental Course for Data Collection

seen even when a vehicle is not approaching . We considered that this result is caused by the fact that fluctuations due to obstacle avoidance behavior and swing of the steering wheel operation exceed a fluctuation due to passing vehicles.

Next, Fig. 4 shows the result of collected sound level data. Similarly, red color highlights show the vehicle approaching period, and there are parts that change greatly. This result might have been influenced by the wind noise generated during running a bicycle.

Finally, Fig. 5 shows frequency spectrogram obtained by applying Fourier transformation to the collected sound data. (a) and (b) in the figure show spectrograms at the time of vehicle approaching state and non-vehicle approaching state, respectively. In the case (a), a spectrum of 2000 [Hz] or less is always stronger than that of the case (b), and a spectrum is strongly increased to about $8000[\mathrm{~Hz}]$ for 2 seconds when the vehicle approaches the closest. From these experimental results, we found that the frequency characteristics of sound

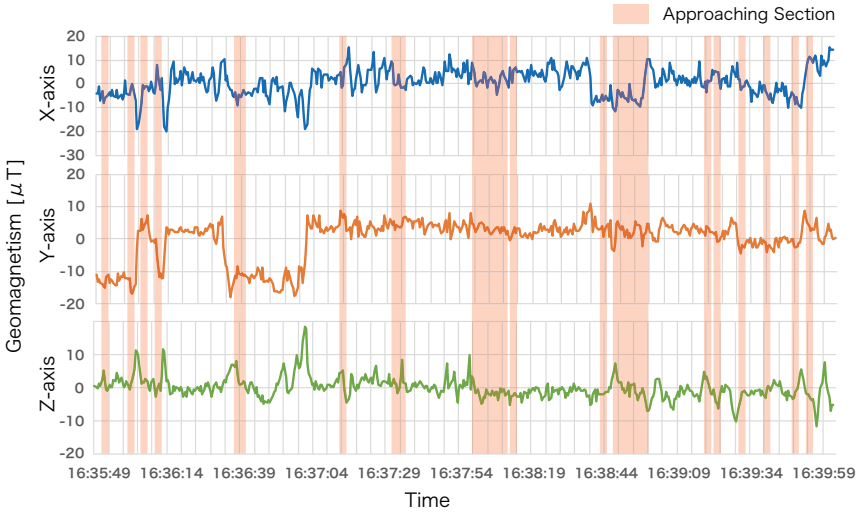

Fig. 3. Result of Geomagnetic Data

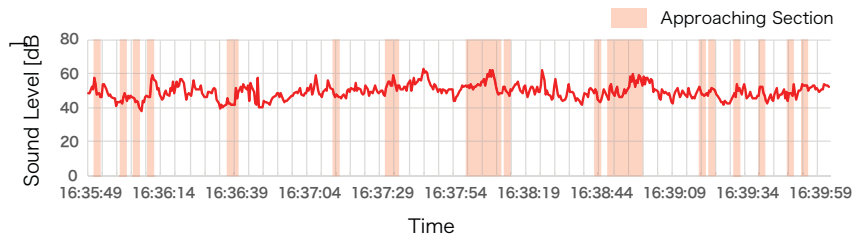

Fig. 4. Result of Sound Level

data are useful to detect an approaching vehicle.

\section{Approaching Vehicle Detection Method using SMARTPHONE}

In this section, first, we present an overview of our proposed approaching vehicle notification system. After that, a training data acquisition method necessary to apply machine learning is explained. Finally, we describe the approaching vehicle detection model trained by machine learning and summarize the evaluation results.

\section{A. Proposed Approaching Vehicle Notification System}

Fig. 6 shows the overall configuration of the proposed approaching vehicle notification system.

This system is composed of a smartphone and notification devices such as a smart glasses or a smartwatch. In the case where the smartphone is fixed to the handlebar of the bicycle with a mounter, it is possible to perform both roles of detection and notification. If a smartphone is in a chest pocket and so on, an additional notification device is necessary to notify an approaching vehicle. Therefore, in consideration of the safety while running bicycle, we use eyeglasses type devices such as TwinkleMegane (Fig. 7 created by Kashimoto et al. [11], FUN'IKI Ambient Glasses [12] and smart watches as notification devices. These can be connected to a smartphone with Bluetooth and notify users by light emission or vibration when a vehicle is detected.

\section{B. Approaching Vehicle Detection Method}

In this system, the approach of a vehicle is identified by machine learning. The application process of machine learning is divided into four processes: (1) Collection of learning data, 


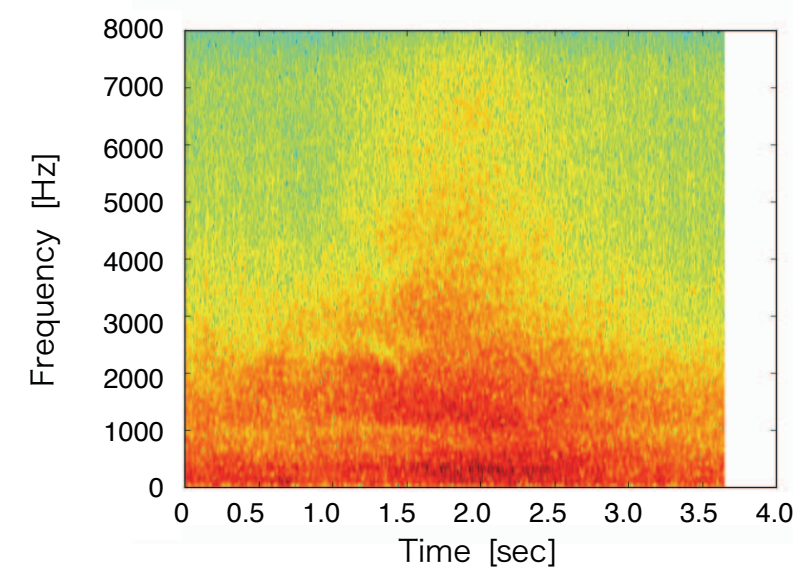

(a)Vehicle Approaching

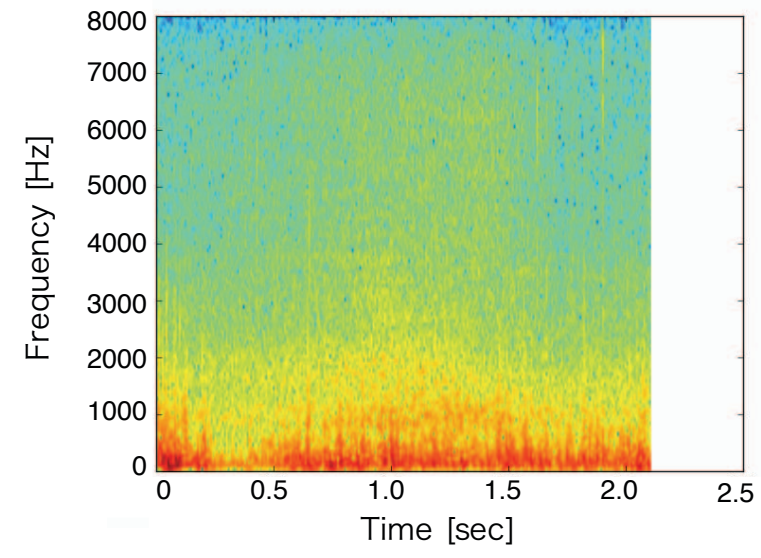

(b) Non-Vehicle Approaching

Fig. 5. Frequency Spectrogram in Each Situation

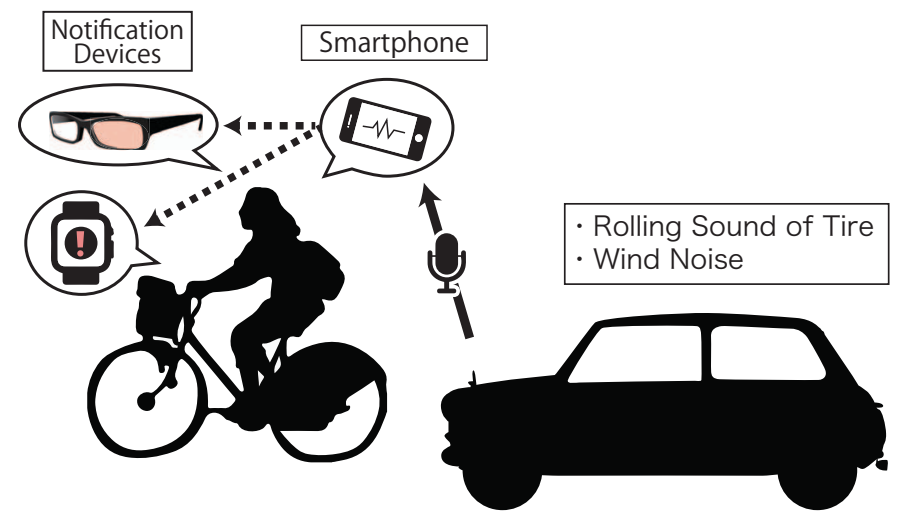

Fig. 6. Configuration Diagram of Proposed System

(2) Generation of training data,(3) Extraction of features, and (4) Construction of approaching vehicle detection model. Each process is described below.

\section{(1) Collection of learning data}

First, we collected sound data as training data. The collection method and recording format are the same as the experimental method described in Section 3.2. The smartphone and the GoPro camera are attached on the handlebars of the bicycle. We performed six round trips at the experimental course shown in Fig. 2, and collected sound data of a total of 34 minutes and 17 seconds. At this time, 158 vehicles passed in total.

(2) Generation of training data

It is necessary to label training data with frequency characteristic which corresponds to approaching a vehicle, in advance. We divided the series of sound data one by one manually into car approaching parts and other parts and labeled vehicle approach (car) and ambient noise (noise), respectively.

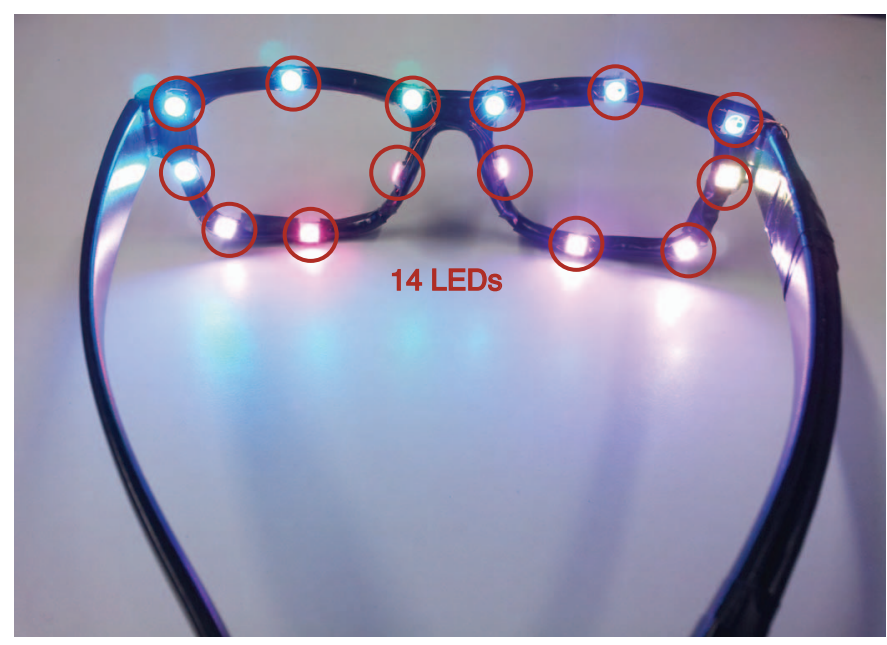

Fig. 7. Twinkle Megane

\section{(3) Extraction of feature}

Features of the frequency spectrum when the vehicle approaches are extracted from the collected data. The sound data are divided into constant time intervals (Time-window). Next, features are extracted from these for each time-window section. Here, we set the size of Time-window to 0.5 seconds (every 8000 samples), empirically. Additionally, frequency spectrum of 20 $8000[\mathrm{~Hz}]$ obtained by performing FFT are divided for every $1.0[\mathrm{~Hz}]$ and these are used as features.

(4) Construction of approaching vehicle detection model A machine learning model is constructed by using the frequency features of the labeled sound data as training data. A data mining tool called "Weka" is used to construct a classifier. In this study, the approach vehicle detection model is constructed using REPTree which 


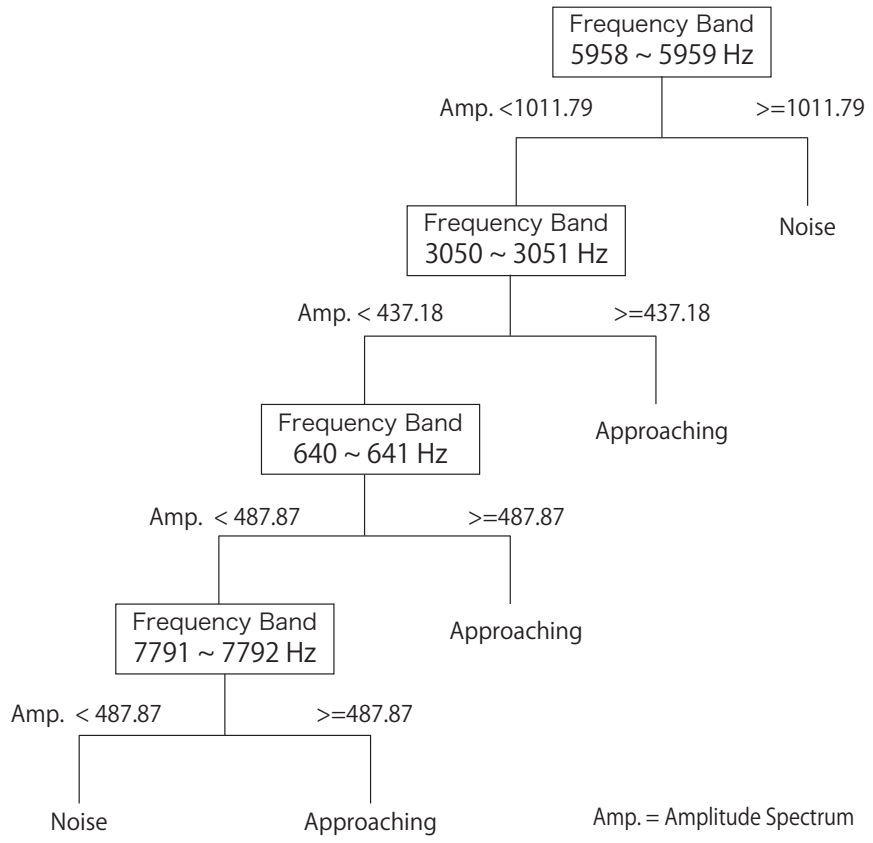

Fig. 8. Approaching Vehicle Detection Model

TABLE II

CONFUSION MATRIX

\begin{tabular}{c|cc}
\hline \hline & Noise & Car \\
\hline Noise & 353 & 23 \\
Car & 5 & 691 \\
\hline
\end{tabular}

creates a shallow hierarchical binary tree, to simplify the implementation to the smartphone application. We used 1072 samples for the training data. The classifier created as a result of learning is shown in Fig. 8.

\section{Evaluation of model}

We applied 10-fold cross validation to all data and evaluated the detection accuracy. Table II and Table III show confusion matrix and the result of evaluation, respectively. Precision is the ratio of correct data that was in actual state among data recognized as "car" or "noise". The recall is the ratio of correctly recognized data as the correct situation among the corresponding sounds. The F-value is a harmonic mean of Precision and Recall and is indicated by the following formula.

$$
F_{\text {value }}=\frac{2 \cdot \text { Recall } \cdot \text { Precision }}{\text { Recall }+ \text { Precision }}
$$

As a result, we found that this classier can identify vehicle approach with high accuracy with an average $\mathrm{F}$ value of 97.4 [\%].

\section{Conclusion}

In this paper, we proposed an approaching vehicle detection method using a smartphone to decrease fatal accidents of elderly people while riding a bicycle. We collected sound data in a real environment and created an approaching vehicle detection model by using machine learning. Finally, as a
TABLE III

THE RESUlT OF EVALUATION

\begin{tabular}{c|ccc}
\hline \hline Label & Precision [\%] & Recal [\%] & F-value [\%] \\
\hline Noise & 98.6 & 93.9 & 96.2 \\
Car & 96.8 & 99.3 & 98.0 \\
\hline Average & 97.4 & 97.4 & 97.4 \\
\hline
\end{tabular}

result of accuracy evaluation with 10-fold cross-validation, we confirmed that it can detect an approaching vehicle at the closest approach with high precision, and an average F-value of 97.4 [\%].

As future work, since this study is aimed at notifying in advance, it is necessary to improve the current model which detects the closest approach. Now, it is necessary to set the target time for advance notification. It is known that the elderly people take about 2 seconds for avoidance behaviors and stop behaviors during running bicycles, according to the survey results of Japan Safe Driving Center [13]. Based on this result, we considered that it is reasonable to make a notification before 5 seconds of the closest approach since these behaviors are performed after proximity notice and recognition, and improve the current model. After that, in order to confirm the optimal notification way and notification timing, we plan to analyze the user's behavior at the time of notification.

Additionally, since the vehicle detection model is trained by using the data, that only collected in a limited environment, we plan to collect data in multiple environments to improve the model that can be used in various environments. Then, it is assumed that other noises such as human voice and railroad noise influence to performance of the vehicle detection system. Therefore, it is necessary to deal with these factors.

Furthermore, we also examine the estimation of other factors such as the direction of the approaching vehicle, the vehicle speed and the type of car, by considering the speed of the self-speed and the Doppler effect.

\section{ACKNOWLEDGEMENT}

This work was supported by JSPS KAKENHI Grant Number $15 \mathrm{H} 05708$.

\section{REFERENCES}

[1] National Police Agency: "Traffic Accidents Situation: 2015," 2015.

[2] Cabinet Office: "Annual Report on the Aging Society: 2016 Chapter 1 Situation of aging," 2016.

[3] Tokyo Metropolitan Government: "Tokyo Bicycle Travel Space Facility Promotion Plan," 2012.

[4] Stephen Smaldone, Chetan Tonde, Vancheswaran K. Ananthanarayanan Ahmed Elgammal and Liviu Iftode: "The Cyber-Physical Bike: A Step Towards Safer Green Transportation," In Proceedings of the 12th Workshop on Mobile Computing Systems and Applications, HotMobile'11, ACM(New York, NY, USA, 2011), pp.56-61, 2011.

[5] Naoki Shimoyama, Hikaru Ando, Kazuto Yamazaki, Takahiro Ishii, Masayuki Hirayama : "A study of bicycle accident prevention system by riding analysis using multi-sensor," SIG Technical Reports, System LSI Design Methodology, 2015-EMB-36(51), pp.1-6, 2015.

[6] Hiroyuki Yoshida, Miyuki Nakano, Midori Sugyaya : "A Study of Accident Prevention both of Bicycle and Pedestrian by Approaching Detection based on mobile GPS," SIG Technical Reports, Ubiquitous computing systems, 2015-UBI-46, pp.1-8, 2015. 
[7] Masaru Takagi, Kosuke Fujimoto, Yoshihiro Kawahara, Tohru Asami: "Detecting hybrid and electric vehicles using a smartphone," Proceedings of the 2014 ACM International Joint Conference on Pervasive and Ubiquitous Computing, New York, USA, pp.267-275, 2014.

[8] Ministry of Internal Affairs and Communications: “ 2015 year Information communication white paper Internet spreading situation Part 3 Basic data and policy trends," 2015.

[9] Shigemi Ishida, Song Liu, Kohei Mimura, Shigeaki Tagashira, Akira Fukuda : "Design and Initial Evaluation of Acoustic Vehicle Coun System utilizing Dynamic Time Warping," SIG Technical Reports, Intelligent Transport Systems, 2016-ITS-64, pp.1-6, 2016.
[10] Bence Koszteczky, Gyula Simon: "Magnetic-based vehicle detection with sensor networks, Instrumentation and Measurement Technology Conference (I2MTC)," pp.265-270, 2013.

[11] Kashimoto Yukitoshi, Aryan Firouzian, Zeeshan Asghar, Goshiro Yamamoto, Petri Pulli: "Twinkle megane: Near-eye LED indicators on glasses in tele-guidance for elderly," IEEE International Conference on Pervasive Computing and Communication Workshops, pp.1-6, 2016.

[12] FUN'IKI Ambient Glasses, http://fun-iki.com/.

[13] Japan Safe Driving Center: "Study on safety behavior etc. of elderly people by traffic mode," 2004 survey research report, pp.22-25, 2005. 